\title{
Arquitetura baseada em Web Semântica para aplicações de Hipermídia Adaptativa
}

\author{
Sandro José Rigo ${ }^{1,2}$, Éverton Eckert Schneider ${ }^{1}$, José Palazzo M. de Oliveira ${ }^{2}$ \\ ${ }^{1}$ Universidade do Vale do Rio dos Sinos (UNISINOS) São Leopoldo - RS - Brasil \\ rigo@unisinos.br, everton.schneider@gmail.com \\ ${ }^{2}$ Instituto de Informática - Universidade Federal do Rio Grande do Sul (UFRGS) \\ Porto Alegre - RS - Brazil \\ palazzodinf.ufrgs.br*
}

\begin{abstract}
This paper describes an architecture for Adaptive Hypermedia applications based on Semantic Web. The main goal of this proposal is to evaluate experimentally the advantages that could be obtained with a semantic based application description and with improved interaction possibilities. The high flexibility to generate adaptations and facilities for semantic content annotation are some of the advantages of this architecture.
\end{abstract}

Resumo. Este documento descreve uma arquitetura para aplicações de Hipermídia Adaptativa baseada em Web Semântica. O principal objetivo da proposta é avaliar experimentalmente as vantagens que podem ser obtidas a partir da descrição da aplicação com recursos de semântica e com possibilidades maiores de interação. Algumas destas são a grande flexibilidade para a geração de adaptações e facilidades para a anotação semântica de conteúdos.

\section{Introdução}

A Internet está em crescimento há vários anos, fato que vêm gerando uma demanda pela personalização de conteúdos das páginas Web, visando evitar a sobrecarga cognitiva [1] existente atualmente. Esta melhoria pode ser obtida com técnicas de Hipermídia Adaptativa, a partir das quais são relacionadas informações sobre o domínio da aplicação com informações sobre o perfil de usuários, o que permite a geração de resultados mais adequados ao contexto de uso para cada usuário ou grupo de usuários.

A Hipermídia Adaptativa aborda o desenvolvimento de sistemas capazes de promover a adaptação de conteúdos e recursos hipermídia oriundos de diversas fontes (banco de dados, documentos na Internet, serviços Web) e apresentados em diversos formatos (por exemplo, texto, áudio, imagens). Esta adaptação se dá em relação às

\footnotetext{
* Agradecimentos. Este trabalho foi parcialmente suportado pelos projetos Pronex FAPERGS, grant 0408933 e CTInfo CNPq, grant 550.845/2005-4. O último autor é parcialmente apoiado pelo CNPq
} 


\section{Simpósio Brasileiro de Sistemas de Informação}

informações conhecidas de um determinado usuário, que descrevem suas preferências, objetivos, conhecimentos prévios e necessidades. É possível encontrar aplicação direta para esta abordagem em áreas como educação, sistemas de informação para ajuda online, comércio eletrônico e marketing, entre outros. O resultado almejado é proporcionar a cada usuário uma interface onde estilo, conteúdo, recursos e hyperlinks são apresentados conforme seus objetivos e preferências [1,2]. Este resultado pode ser obtido com técnicas como a apresentação adaptativa ou a navegação adaptativa, com o objetivo de adaptação de conteúdo e de adaptação de hyperlinks, respectivamente.

Apesar de serem conhecidos estudos sobre área de Hipermídia Adaptativa desde a década de 90 [3], alguns trabalhos mais recentes sinalizam uma falta de padrões para a área, o que pode levar a problemas de interoperabilidade. Estes podem ser solucionados com o uso da Web Semântica, cujo papel é permitir que sistemas computacionais possam realizar melhor uso dos dados presentes na Web [4], a partir da aplicação de ontologias, com linguagens como OWL ${ }^{2}$. Ontologias são usadas como uma forma de representar conhecimento sobre domínios diversos e descrevem normalmente indivíduos (instâncias), classes (conjuntos, coleções, tipos de objetos), atributos (propriedades, características), relações e eventos (mudança de atributos ou relações) [5, 6]. Além de ontologias a Web Semântica permite a descrição de recursos, por exemplo, com a linguagem $\mathrm{RDF}^{3}$, que provê uma especificação padrão para descrição de metadados sobre recursos na Web.

Em geral as aplicações de Hipermídia Adaptativa são descritas por modelos que indicam, por exemplo, informações do domínio, apresentação de conteúdos ou características de usuários. As adaptações são geradas com base em regras e na aquisição de informações do usuário. A utilização dos recursos da Web Semântica neste contexto possibilita a descrição de modelos mais flexíveis e abrangentes, de modo a facilitar a tarefa de adaptação [7, 8]. Entretanto uma dificuldade observada na aplicação de recursos de Web Semântica é a inerente necessidade de especialização dos usuários responsáveis pela geração das relações, o que torna proibitivo a utilização em larga escala, por exemplo, de editores de ontologias ou a anotação semântica.

Este trabalho descreve uma arquitetura para aplicações de Hipermídia Adaptativa que incorpora recursos semânticos. A utilização das tecnologias da Web Semântica permite maior flexibilidade na descrição dos modelos para domínio da aplicação e apresentação de conteúdos. A proposta também facilita a interação dos usuários responsáveis pela geração de conteúdo $\mathrm{Web}$, de modo que estes não precisem de conhecimentos profundos sobre tecnologias ou linguagens de desenvolvimento para Internet e nem sobre publicação de conteúdo na Web ou sobre Web Semântica. Deste modo um dos principais diferenciais do trabalho consiste na abordagem privilegiando o usuário responsável pela criação e manutenção do conteúdo, evitando a necessidade de conhecimento aprofundado da área de Web Semântica. O outro diferencial do trabalho consiste em uma abordagem simplificada para a descrição e utilização de modelos necessários para a geração de adaptações. Foi utilizado um conjunto de ontologias que permite a descrição da estrutura do site Web e os formatos gerais de apresentação de conteúdos, que podem ser relacionados a partir de regras baseadas no comportamento e

\footnotetext{
${ }^{2}$ http://www.w3c.org/owl

${ }^{3}$ http://www.w3c.org/rdf
} 


\section{Simpósio Brasileiro de Sistemas de Informação}

nas informações do perfil dos usuários. Por fim, o trabalho foi desenvolvido de forma que as adaptações não necessitem da identificação pessoal dos usuários, em uma abordagem onde a identificação de classes de comportamento a partir das sessões típicas dos usuários origina a informação necessária para as adaptações. Todo o processamento destas informações é realizado continuamente e integrado ao funcionamento do sistema, sem necessidade de etapas adicionais para geração de padrões.

\section{Análise de trabalhos relacionados}

Vários sistemas adaptativos foram desenvolvidos sem a utilização de recursos de semântica e baseiam-se em modelos e regras. Nestes casos não existem possibilidades mais flexíveis em relação à geração das adaptações, como o uso de inferências ou o compartilhamento de informações (por exemplo, do perfil de usuários). $\mathrm{O}$ AHA(Adaptive Hypermedia Architecture) [9] mantém um modelo de usuários baseado em conhecimento sobre conceitos e oferece recursos de adaptatividade de conteúdo e hyperlinks através do uso de fragmentos inseridos no código $\mathrm{HTML}^{4}$. O sistema Elena PLA [10], com foco principal no suporte para personalização em ambiente distribuído, utiliza modelo de usuários, repositórios de conteúdos, serviços de anotação e de recomendação.

A aplicação da linguagem $\mathrm{XML}^{5}$ para a descrição de estruturas e conteúdos, indicações de anotações ou descrição de perfil de usuários pode ser acompanhada a partir de exemplos como AdaptWeb [11], ambiente adaptativo para aprendizagem, onde são observados o programa de aprendizado, o conhecimento do aluno e suas preferências de interação. Nele pode-se observar a interação de recursos como a anotação semântica, a descrição de estrutura de conteúdos e a adaptação de características dos conteúdos, de acordo com o contexto do usuário. Um ambiente de autoria permite a descrição de metadados associados ao material sendo publicado e uma descrição do modelo do usuário é utilizada a partir de uma ontologia, prevendo operações de personalização.

Outros sistemas baseados em Web Semântica foram desenvolvidos a partir de abordagens inicialmente descritas sem propósito de adaptação, tal como a proposta do método ASHDM (Adpative Semantic Hypermedia Design Method) [15], descrito a partir do SHDM (Semantic Hypermedia Design Method) [12], método de modelagem que busca representar tanto um projeto conceitual como projeto navegacional utilizando linguagens de descrição de ontologias, possibilitando o desenvolvimento de sites Web a partir de características semânticas.

Alguns exemplos tratam da integração de recursos de Web Semântica e Hipermídia Adaptativa. O OntoWeaver [13] é uma proposta baseada em ontologias para desenvolvimento de sites na Web, incluindo funcionalidades de adaptação. Utiliza-se uma ontologia de visualização, para modelar a estrutura de páginas Web e a interface do usuário, e uma ontologia de apresentação, definida para descrever os estilos de apresentação e os formatos para as páginas [14]. Realiza a adaptação de informações com um modelo de usuários e um modelo de customização baseados nas preferências de

\footnotetext{
${ }^{4}$ http://www.w3c.org/MarkUp

${ }^{5}$ http://www.w3c.org/XML
} 


\section{Simpósio Brasileiro de Sistemas de Informação}

cada usuário. O OntoWebber [16] utiliza ontologias para composição da interface a partir dos seguintes elementos: "cards", "links" e "pages". Um elemento "page" integra os demais elementos, que podem ser dinâmicos, utilizando mecanismo de inferência TRIPLE [17]. A modelagem de personalização permite a identificação de recursos adequados para grupos ou para usuários, com características e propriedades indicando interesses e condições de navegação desejadas. A proposta do SEAL [18] também é baseada em ontologias e voltada para a descrição de portais Web. As etapas para a construção destes portais Web são o projeto de ontologia, integração de dados, projeto de site Web e implementação. O projeto de site envolve a construção do modelo de navegação, modelo de entrada e modelo de personalização. Para sua implementação é utilizado o framework $\mathrm{KAON}^{6}$, que possui ferramentas integradas para a descrição de ontologias, integração de dados e geração personalizada do portal, a partir da identificação de usuários e seu contexto.

Desta forma, observa-se que a utilização de ontologias para a descrição de estruturas, modelos e informações do perfil de usuários facilita a geração de adaptações e sua manutenção. Entretanto estas construções tendem à geração de uma estrutura de difícil manuseio pelos usuários responsáveis pela edição dos conteúdos. Em geral as adaptações são geradas com base no conhecimento de preferências de usuários, demandando a sua identificação.

\section{Detalhamento da arquitetura desenvolvida}

A arquitetura proposta tem como objetivo apresentar aos usuários páginas com características adaptativas em um ambiente semântico. Foi tomada como premissa a geração de adaptações sem a identificação do usuário. Para isso as informações de uso são aproveitadas como base, junto com informações de perfis de grupos de usuários obtidos com o acompanhamento do uso. São empregadas duas ontologias, uma contendo informações e hierarquia das páginas pertencentes ao site Web (Ontologia da Aplicação) e a outra com a definição da interface gráfica que cada página pode possuir (Ontologia de Apresentação). O sistema mantém uma base de dados para os conteúdos das páginas, que armazena todos os conteúdos em formato textual, relacionando-os a partir da Ontologia da Aplicação e permitindo representar diferentes conteúdos para uma mesma página, com informações que podem ser usadas na tarefa de adaptação. Todos os acessos dos usuários são mantidos de forma resumida em uma base de dados. As regras de adaptação baseiam-se na utilização do site e na estrutura descrita nas ontologias. Elas relacionam informações do perfil do usuário com a estrutura do site, indicando possibilidades de adaptação utilizadas em conjunto com informações descritas na Ontologia de Apresentação. As possibilidades de adaptação implementadas foram definidas como acréscimos à estrutura do site, na forma de hyperlinks adicionais, e como ajustes de conteúdos, na forma de exibição de material adicional.

Para proporcionar flexibilidade na implementação da arquitetura, a mesma foi separada em dois módulos: validação e adaptação. Seu funcionamento geral, descrito na figura 1, pode ser resumido como: recebimento de solicitação de acesso e gravação da informação correspondente ao modelo do usuário; consulta da página solicitada na Ontologia da Aplicação; consulta do template correspondente na Ontologia de

\footnotetext{
${ }^{6} \mathrm{http}: / /$ kaon.semanticweb.org/
} 


\section{Simpósio Brasileiro de Sistemas de Informação}

Apresentação; montagem do resultado intermediário (ainda sem adaptação) em código HTML; verificação do padrão comportamental através do modelo do usuário; aplicação de regras de adaptação; geração do código final (com adaptações) em HTML; envio da resposta para o usuário.

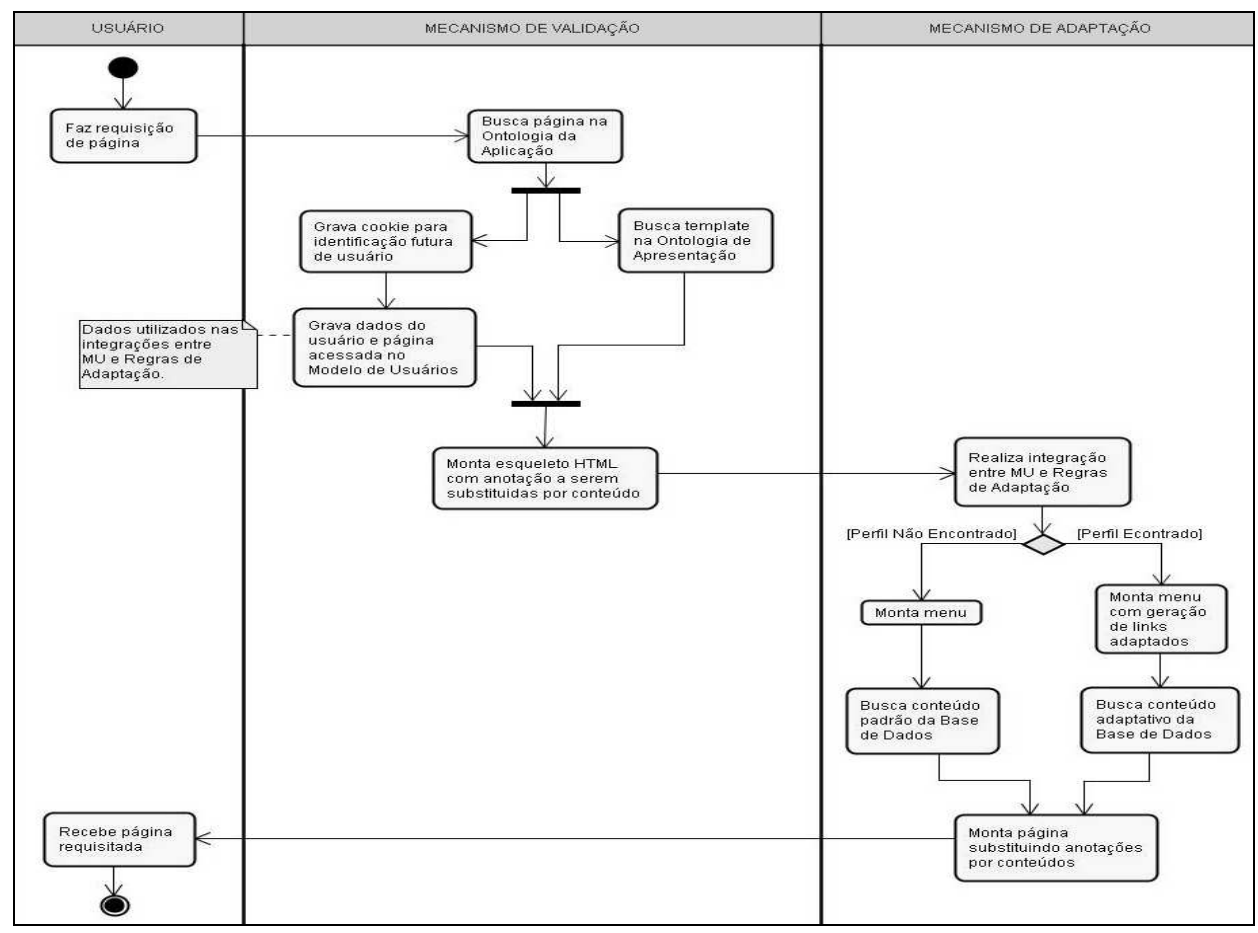

Figura 1 - Funcionamento geral da arquitetura desenvolvida

O mecanismo de validação é responsável pela montagem inicial do código HTML da página requisitada e pela gravação de informações que possibilitam adaptações de conteúdo em requisições futuras. Utiliza-se um cookie para identificar acessos da sessão de um usuário. Esta abordagem foi escolhida por facilitar a identificação de informações da sessão, apesar de sua desvantagem no caso de desativação junto ao navegador do usuário. Cada acesso gera um registro que compõe o modelo de usuários, contendo dados sobre a navegação, tais como a página acessada e o tipo de conteúdo. Algumas destas informações são geradas pela requisição recebida e outras são complementadas com uma consulta à ontologia da aplicação. Nela estão descritas a estrutura da aplicação e os detalhes de cada página, permitindo a categorização do acesso com informações semânticas como o tipo de conteúdo e as relações desta página com as demais.

Estas ontologias foram descritas manualmente por especialistas no domínio da aplicação, com uso do editor de ontologias Protege ${ }^{7}$, tendo sido utilizada a linguagem OWL para sua representação. Parte da Ontologia da Aplicação está descrita brevemente na figura 2, na qual podem ser observadas algumas relações, como a "possuiSubpagina". Além destas relações são mantidos alguns atributos definidos nesta ontologia, como

$7 \mathrm{http}: / /$ protege.stanford.edu 


\section{Simpósio Brasileiro de Sistemas de Informação}

"tipoConteudo" e "template", e outros reutilizados de outras terminologias conhecidas, como o Dublin Core ${ }^{8}$, no caso de "dc:description" e "dc:creator".

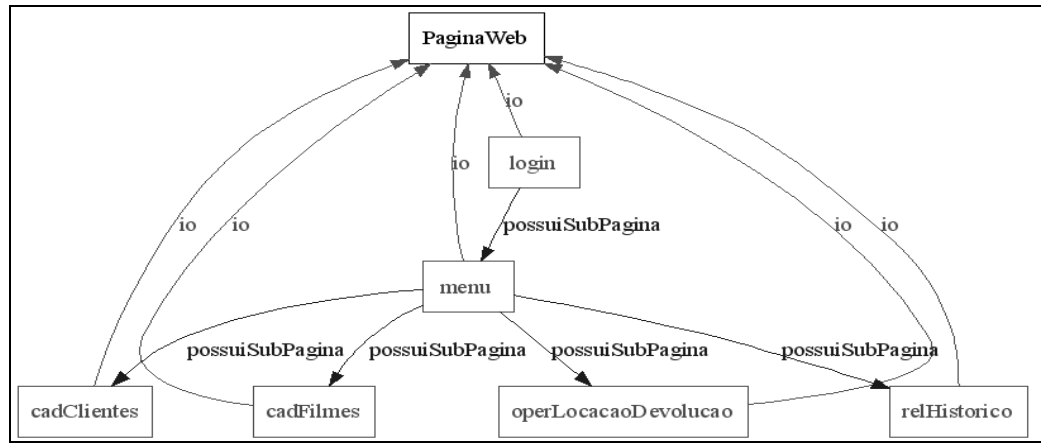

Figura 2 - Trecho da estrutura da Ontologia da Aplicação

A Ontologia de Apresentação descreve características da interface a ser definida. Cada elemento desta ontologia indica especificamente um tipo de formato, no elemento "template". Estes formatos são descritos, na ontologia de apresentação, a partir dos conceitos "template", "elemento" e "sub-elemento", que permitem a descrição de dados da página e possibilitam a geração do código HTML básico com anotações de conteúdo para substituições. Nas seções onde devem ser feitas substituições por conteúdos adaptados são utilizados marcadores específicos. A figura 3 ilustra a descrição de uma típica área de interface, descrita como "TPL01conteudo" e composta por elementos com informações para a geração do HTML ("tag") e informações para substituição (“swah:text”). Estas informações são utilizadas em conjunto com outros elementos da codificação padrão necessária para a montagem de uma página em HTML.

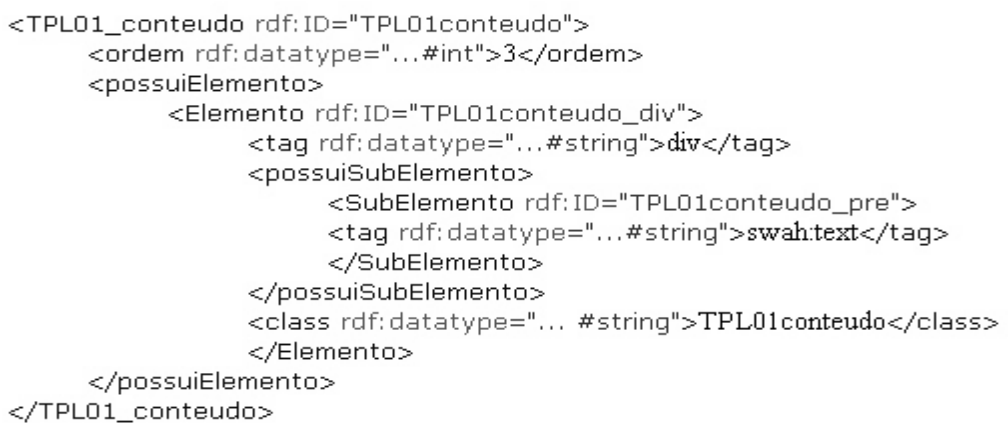

Figura 3: Parte da ontologia de apresentação

O mecanismo de adaptação realiza a análise da codificação HTML gerada pelo mecanismo de validação e substitui suas anotação por conteúdos. Para determinar os conteúdos correspondentes a cada anotação é feita uma análise entre o modelo de usuário e as regras de adaptação, em busca de um padrão adequado. As regras são descritas a partir de condições e ações. As condições correspondem às informações do comportamento percebido do usuário e as ações correspondem à utilização do conteúdo para substituições. Estas regras são geradas pelo acompanhamento do comportamento

\footnotetext{
${ }^{8}$ http://dublincore.org/
} 


\section{Simpósio Brasileiro de Sistemas de Informação}

dos usuários do site Web. Foram definidos três tipos de regras, para o menu de navegação, associadas ao perfil do usuário e ao conteúdo. As regras de menu são as mais simples e são compostas de uma condição indicando a página acessada e diversas ações, que correspondem às páginas sugeridas em hyperlinks junto ao menu gerado pela aplicação. As regras associadas ao perfil do usuário são descritas com condições onde são indicadas as páginas mais acessadas por determinado grupo de usuários. As regras de conteúdo integram informações do perfil do usuário, página acessada e conteúdo a ser adaptado. São compostas de condições associadas ao tipo de perfil e à página acessada. As ações são associadas às áreas da interface que possibilitam adaptação, como por exemplo, o topo da página, o conteúdo principal e a área destinada a conteúdos complementares. A definição do tipo de perfil do usuário leva em consideração o número de vezes que cada página associada às regras que definem este perfil foi acessada. A geração do perfil pode ser feita de forma manual, com a edição de regras que o definem, ou de forma semi-automática, com a análise das sessões dos usuários. A cada perfil de usuário definido correspondem um grupo com comportamentos similares e são associadas sugestões de hyperlinks para o menu e a substituição de conteúdo por informações adaptativas. Caso nenhuma regra válida seja encontrada, a página a ser apresentada ao usuário será montada com seu conteúdo padrão.

Para facilitar a criação e publicação de páginas por usuário sem conhecimentos em tecnologias da Web Semântica, foi desenvolvida uma ferramenta de administração de conteúdos criados através da arquitetura, denominada SWAH (Semantic Web / Adaptive Hypermedia). A interface disponibiliza telas de cadastros de páginas e inserção de conteúdos, telas para upload de imagens e regras, além de telas de configuração geral dos parâmetros do ambiente. A Figura 4 exibe algumas telas do ambiente de administração.
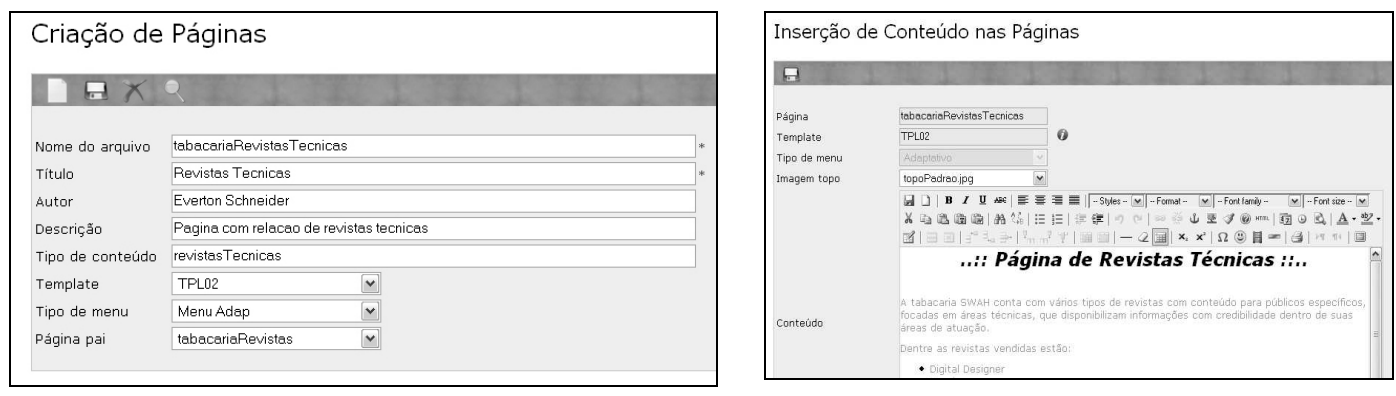

Figura 4: Telas da interface SWAH

$\mathrm{O}$ item à esquerda da Figura 4 apresenta a tela de criação de páginas, que mantém a Ontologia da Aplicação atualizada com cada página atualizada pelo usuário. Nesta tela constam informações de cadastro como nome do arquivo, título da página, autor, descrição e tipo de conteúdo, template escolhido, tipo de menu a ser utilizado (adaptativo, inferido ou fixo) e página superior na hierarquia (página “pai”). Após o cadastro de uma página é necessário inserir conteúdo para a mesma. $\mathrm{O}$ item à direita da Figura 4 mostra a tela de inserção de conteúdos, onde é editado o conteúdo padrão da página, ou seja, conteúdo que é apresentado caso não existam regras de adaptação para a mesma. Nesta tela podem ser observadas as informações gerais obtidas da tela anterior, no cadastro da página, seguida pela área de edição do conteúdo onde está disponível 


\section{Simpósio Brasileiro de Sistemas de Informação}

uma barra de ferramentas que busca facilitar a interação entre os usuários e a edição de páginas. No caso da inserção de conteúdo adaptativo, além destas informações é indicado um nome para o tipo de conteúdo da página (que também deverá ser usado na montagem das regras), bem como inserido o conteúdo correspondente. Os dados cadastrados pela interface SWAH são armazenados em um banco de dados MySQL ${ }^{9}$, sendo que o acesso às ontologias é realizado com uso da biblioteca $\mathrm{RAP}^{10}$, sendo utilizada a linguagem PHP $5^{11}$ para a implementação dos sistema.

\section{Análise da experimentação}

Para a aplicação das funcionalidades implementadas na arquitetura foi desenvolvido um caso de uso com base nos requisitos para uma tabacaria, possuidora de três categorias de produtos: livros, revistas e DVDs. Devido à facilidade de relacionamento de áreas entre essas categorias, foram feitos testes com regras para sugestão de hyperlinks no menu de páginas distintas e com a substituição ou complementação de conteúdos. Para o teste foram criados quatro perfis de usuários, identificados como adulto, jovial, infantil e técnico. A primeira etapa para a criação do site foi o cadastro de suas páginas, tendo sido criadas dezessete páginas, todas relacionadas às três categorias de produtos citadas. Foram criadas várias subcategorias para distinguir os tipos de produtos, como por exemplo, livros técnicos, revistas infantis, DVDs de filmes, entre outros. A seguir foram inseridos os conteúdos das páginas e criados conteúdos adaptativos.

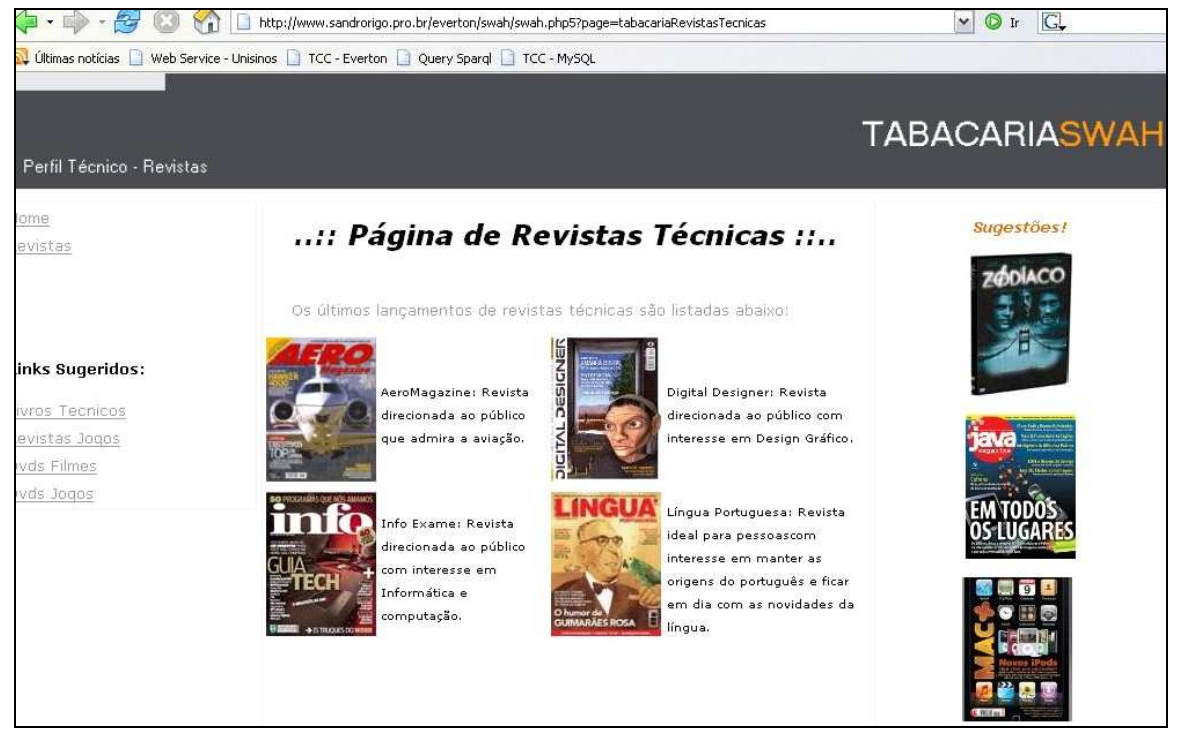

Figura 5: Página com conteúdos adaptados

O exemplo apresentado na figura 5 é de uma página de revistas técnicas. Essa página teve todas suas áreas adaptadas devido às regras de adaptação e o comportamento do usuário em sua navegação entre as páginas do portal, o que resultou na avaliação de seu perfil como perfil técnico. Segundo as regras criadas para a o site de

\footnotetext{
${ }^{9}$ http://www.mysql.com/

${ }^{10} \mathrm{http}: / /$ sites.wiwiss.fu-berlin.de/suhl/bizer/rdfapi/

${ }^{11} \mathrm{http}: / /$ www.php.net/
} 


\section{Simpósio Brasileiro de Sistemas de Informação}

experimentação, um usuário de perfil técnico acessa com maior freqüência categorias como livros de auto-ajuda e livros técnicos, revistas de filmes, jogos e de assuntos técnicos, além de DVDs de jogos. Estas informações podem ser observadas na figura 6 , onde a regra descrita com ao atributo type='profile' e name='tecnico' descreve estas possibilidades de adaptação.

As regras cadastradas podem ser verificadas na Figura 6. É importante observar a indicação dos conteúdos adaptativos nas regras com atributo type='content'. A regra indica que se o usuário possui o perfil técnico e estiver acessando a página com nome "tabacariaRevistasTecnicas", o topo deve usar o conteúdo "topRevTecnicasPT", a área central o conteúdo "dcRevTecnicaPT" e a área lateral o conteúdo "ocRevTecnicaPT". Esses nomes são atribuídos aos conteúdos na sua criação, através da interface de administração, na tela de inserção de conteúdos adaptativos. Também é possível ver na Figura 5 a adaptação de hyperlinks do menu de acordo com as regras anotadas com atributo type='menu'. As regras de menu indicam que, ao acessar a página com nome "revistasTecnicas", sejam mostrados os hyperlinks das páginas de livros técnicos, revistas de jogos, DVDs de filmes e DVDs de jogos.

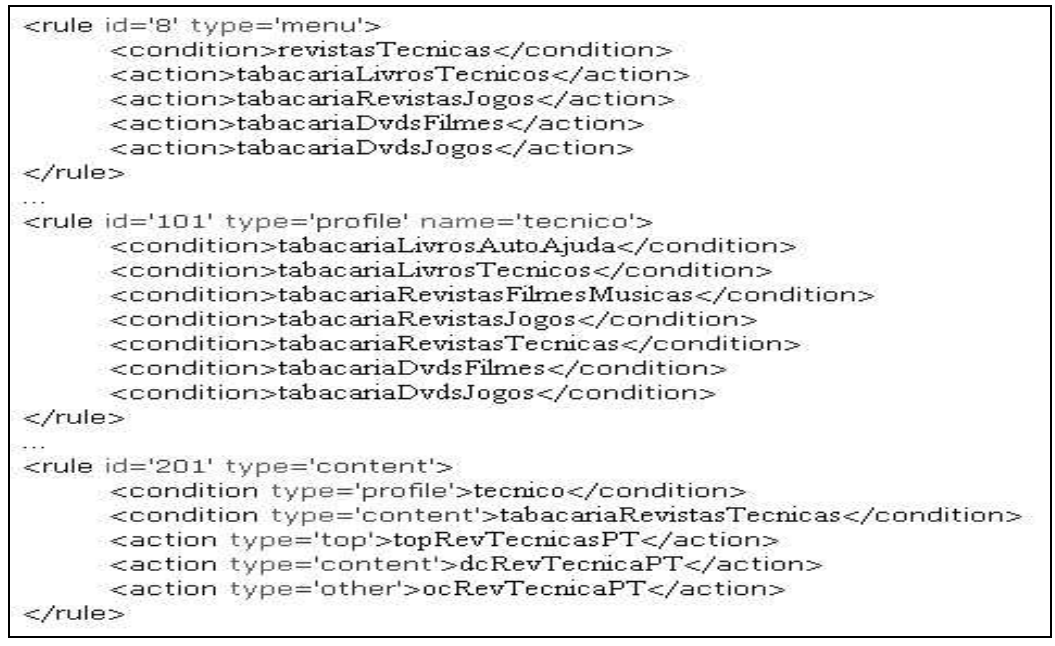

Figura 6: Regras para adaptação de conteúdo

A arquitetura SWAH possibilita esse tipo de comportamento para qualquer página criada com base em sua estrutura, utilizando suas ontologias e regras de adaptação.

\section{Conclusões}

O sistema apresentado permite a descrição de um site web a partir de dois modelos armazenados em ontologias e implementa o acompanhamento de sua utilização. Este acompanhamento possibilita a utilização de regras de adaptação, baseadas na informação semântica descrita nas ontologias. Assim é possível a geração de adaptações de estrutura e de conteúdo, sem necessidade de identificação de usuários individualmente, a partir de tarefas realizadas em uma sessão do usuário. Além disso, o sistema permite ao usuário responsável pela geração e manutenção de conteúdo um acesso facilitado e não requer o conhecimento de conceitos de Web Semântica, o que facilita a sua adoção. 


\section{Simpósio Brasileiro de Sistemas de Informação}

Foi realizada a utilização do sistema para a montagem de um site Web e a realização de testes. A partir desta experiência foram comprovadas as facilidades de edição de conteúdos e também a efetividade das adaptações.

Alguns trabalhos futuros estão sendo encaminhados, no sentido de proporcionar maiores facilidades à edição de conteúdos. A edição de regras de adaptação também está sendo melhorada para que seja facilitada e acrescentada ao módulo de edição de conteúdo. Por fim, a descrição dos formatos de tela deve ser gerada a partir de uma ferramenta mais amigável, baseada em interface Web, de modo a completar o ciclo de ferramentas para que o sistema possa ser completamente utilizado por usuário sem conhecimento em Web Semântica ou desenvolvimento Web.

\section{Referências}

[1] Brusilovsky, P., Karagiannidis, C., and Sampson, D. (2004) Layered evaluation of adaptive learning systems. International Journal of Continuing Engineering Education and Lifelong Learning 14 (4/5), 402 - 421.2004.

[2] De Bra, Arroyo, L., Chepegin, V., The next big thing: adaptive web-based systems. Journal of Digital Information, $\mathrm{V}(5) \mathrm{N}(1) .2004$.

[3] Brusilovsky, P. “Adaptive hypermedia”. User Modeling and User-Adapted Interaction, 11:87-110, 2001.

[4] Kravčík, M., Gašević, D.. Adaptive hypermedia for the semantic web. Conference on Hypertext and Hypermedia. Pages 3-10, Odense, Dinamarca, 2006.

[5] Freitas, F. L. G., "Ontologias e a Web Semântica”. XXIII Congresso da Sociedade Brasileira de Computação. JAI. Campinas, São Paulo, Junho de 2003.

[6] Fensel, D. Ontology-Based Knowledge Management. IEEE Computer, 35(11) pages 56-59, 2002.

[7] De Bra, Arroyo, L., Chepegin, V., The next big thing: adaptive web-based systems. Journal of Digital Information, V(5)N(1). 2004.

[8] Aroyo, L., et al. Ontology-based personalization in user-adaptive systems. 2nd International Workshop on Web Personalization, Recommender Systems and Intelligent User Interfaces (WPRSIUI'06), 2006.

[9] BRA, Paul De, CALVE, Licia. AHA: A Generic Adaptive Hypermedia System. 2nd Workshop on Adaptive Hypertext and Hypermedia, Pittsburg, EUA, 20/06/1998 - 24/06/1998.

[10] Dolog, P. et al. Personalization in distributed e-learning environments. In Proceedings of WWW2004 - The Thirteen International World Wide Web Conference, New York, May 2004. P.85-94. ACM Press.

[11] Oliveira, J. P. M., Muñoz, L., "Adaptative web-based courseware development using metadata standards and ontologies”. CAise conference, Riga, Latvia, June 7-11, 2004.

[12] LIMA. Fernanda. Modelagem semântica de aplicações na WWW. Rio de Janeiro: PUC, Departamento de Informática, 2003.

[13] Ontoweaver - An ontology based approach to web site design and development. Disponível em: <http://kmi.open.ac.uk/projects/akt/ontoweaver>. Acessado em: 14 jun 2007.

[14] LEI, Y., MOTTA, E., DOMINGUE, J.. Modelling Data-Intensive Web Sites with OntoWeaver. In Proceedings The International Workshop on Web Information System Modelling (WISM 2004), pages pp. 106-121, Riga, Latvia.

[15] Assis, P. S., Schwabe, D. Nunes, D. A.,: ASHDM - Model-Driven Adaptation and Meta-adaptation. Adaptive Hypermedia and Adaptive Web-Based Systems, 4th International Conference, AH 2006, Dublin, Ireland, June 21-23, 2006, Proceedings. Lecture Notes in Computer Science 4018 Springer 2006.

[16] Jin, Y., Xu, S., and Decker, S. Ontowebber: Model-driven ontology-based web site management. (SWWS 2001), pages 529-547. Stanford University.

[17] Sintek, M. and Decker, S. (2002). Triple - an rdf query, inference, and transformation language. In First International Semantic Web Conference (ISWC 2002), volume 2342 of Lecture Notes in Computer Science, p. 364-378. Springer.

[18] Maedche, A., Staab, S., Stojanovic, N., Studer, R., and Sure, Y. (2003). Semantic portal: The seal approach. In Spinning the Semantic .pages 317-359. MIT Press. 